\title{
EVOLUCIÓN DE LA LÍNEA I+D EN INFORMÁTICA EDUCATIVA DE LA UNIVERSIDAD EAFIT
}

\author{
Claudia María Zea Restrepo* \\ María del Rosario Atuesta Venegas ${ }^{* \star}$ \\ John Trujillo Vargas
}

\begin{abstract}
The present article make a count of those targets, objectives, theories and projects that have given continued life to the group of Research and Investigation in Educative Computer Aplication at the University Eafit, and how its management and projection have stayed within the scheme of promotion of significant approaches of the technology to the learning environments.
\end{abstract}

\section{RESUMEN}

El presente artículo pretende hacer un recuento de aquellos lineamientos, objetivos, teorías y proyectos que han dado vida continuada al grupo de Investigación y desarrollo en informática educativa de la Universidad Eafit, y cómo su gestión y proyección se ha mantenido dentro del esquema de promover un acercamiento significativo de las tecnologías a los ambientes de aprendizaje.

Palabras clave: ambientes tecnológicos de aprendizaje, ambientes virtuales de aprendizaje, informática y educación, modelos de incorporación de tecnologías, telemática educativa, didácticas apoyadas con TICs.

\section{Introducción}

Este grupo de investigación, que inicia sus labores por el año de 1985, parte de un contexto mundial, en el cual el acelerado ritmo del desarrollo de la ciencia y la tecnología, delinea disciplinas que permiten estudiar el proceso educativo desde ópticas diferentes, trayendo consigo el surgimiento de las tecnologías de la información y la comunicación en los espacios educativos, y cuya resonancia, tanto social como cultural determinan juicios de valor, como la baja calidad de la educación y lo poco pertinente del aspecto científico y tecnológico del sistema educativo en Colombia. En este contexto, la Universidad Eafit, decide promover la investigación del potencial de las tecnologías de información y comunicaciones, y su aplicabilidad en los sistemas de enseñanza, en busca del logro de sus propósitos institucionales, que apuntan a:

\footnotetext{
*czea@eafit educo Universidad EAFIT. Medellín, Colombia. ${ }_{* \star \star}^{* *}$ matuesta@eafit.edu.co Universidad EAFIT. Medellín, Colombia.

jtruji@eafit.edu.co Universidad EAFIT. Medellín, Colombia.
} 
preservar la excelencia en sus procesos de enseñanza, aprendizaje, investigación y proyección social; alcanzar la formación integral de sus estudiantes para que participen positivamente en el desarrollo de la Universidad, del país y de América Latina; desarrollar una interacción dinámica con los sectores empresariales, gubernamentales y académicos, de carácter nacional e internacional y desarrollar la capacidad intelectual de sus alumnos y profesores en todos los programas académicos, con la investigación como soporte básico (Ver http://www.eafit.edu.co/ infoedu).

\section{Cómo iniciamos}

En la búsqueda persistente de encontrar la manera apropiada de incorporar estas tecnologías a los procesos de enseñanza, y de manera que facilitaran a los alumnos alternativas variadas de descubrir e interiorizar nuevos conocimientos, se inician investigaciones y desarrollos en éstas alrededor de los sistemas tutoriales, los cuales se plantean como apoyo a las cátedras y los laboratorios de ingeniería. El primer sistema tutorial que se desarrolla es el "Curso de estática asistido por computador", utilizado por los profesores y estudiantes de los primeros semestres de ingeniería civil, y que llevó a la formalizar una línea de investigación y desarrollo en informática educativa en el interior de la Universidad Eafit en 1985. Esta línea buscó desde sus inicios investigar sobre las posibilidades de la incorporación de la informática en los procesos formales de aprendizaje en el ámbito universitario y de apoyar el logro de objetivos cognitivos de los alumnos de nivel superior.

El enfoque de aquel momento inicial, estaba orientado a transmitir la información con el apoyo de oque se denominaba sistemas CAI (Computer Aid Instruction). El estudiante debía tomar decisiones que eran diagnosticadas por el sistema, el cual entregaba como resultado de este proceso una evaluación de tipo sumativa. El supuesto de aprendizaje estaba apoyado en las conductas del estudiante, en un formato declarativo inserto en el modelo de Gagné (1971). En este patrón se presentaban señales de tipo estimulorespuesta, encadenamientos a través de las respuestas dadas, asociaciones comunicativas, discriminación múltiple, aprendizaje de conceptos y de principios, hasta lograr la acción respuesta solución del problema. El modelo aún se mantenía dentro de un contexto epistemológico conductista, empírico e inductivo, validándose alrededor del cumplimiento de los prerrequisitos lógicos, aunque sólo era capaz de garantizar el cómo, no así el por qué, ni la comprensión de lo aprendido.

De este primer modelo se evoluciona a un modelo cognitivo, enmarcado dentro del procesamiento de información (STI o Sistemas Tutoriales Inteligentes) con algunos cruces y coincidencias con el modelo de aprendizaje de Ausubel (Trujillo, en prensa). La secuencia de aprendizaje se presentaba subordinada a estructuras cognitivas ya existentes, alrededor de una progresión estructurada bajo taxonomías jerárquicas apoyadas en transiciones conceptuales cada vez más complejas, propiciando a su vez procesos de transferencia, integración, reorganización de relaciones y equivalencias de los conceptos, en torno al principio de inclusión ausubeliano. La computadora funcionaba más como agente constructor del modelo de conocimiento (con el apoyo de los diferentes sistemas expertos) que de simple mediador de procesos de comunicación. Este agente, además del modelo de conocimiento en sí, alternaba entre actividades de diagnóstico y actividades didácticas. El modelo como tal se quedaba fundamentalmente en una acción receptiva del estudiante, enfatizando la asimilación de los conceptos estructurantes más que el desarrollo de habilidades sobre los procesos que se presentaban en contextos estáticos reducidos. 
A partir de los cambios de orientación que se operan en los años 1993, hasta el presente, se gestiona el desarrollo cognitivo del estudiante a partir del desarrollo de habilidades de búsqueda y manejo de información para lograr el planteamiento y solución de problemas, apoyadas en habilidades comunicativas y trabajo en equipo. Los problemas-proyectos se plantean orientados a contextos de globalización cultural, teniendo en cuenta los elementos motivadores individuales y a su vez, elaborados conjunta y consensuadamente entre los diferentes miembros de cada grupo, prestando especial atención a la mejora del autoconcepto del aprendiz. Para lograr esto, las diferentes propuestas se han respaldado en los siguientes marcos estratégicos de instrucción:

\section{Dentro del marco piagetiano}

Se desarrolla un acercamiento de tipo constructivista en la fase de exploración, para reforzar la experiencia física a partir del desequilibrio cognitivo; en la fase de invención, para reforzar el intercambio social a partir de la búsqueda de equilibrio en forma interactiva; y en la fase de aplicación, para reforzar la consolidación conceptual a través de la validación de los diferentes contextos en forma grupal (Piaget, 1978).

\section{Dentro del marco de las inteligencias prácticas y enseñanza para la comprensión}

El primer modelo inspirado por Robert Sternberg (teoría triárquica de la inteligencia) y Howard Gardner (inteligencias múltiples) (Gardner et al, 1996), el segundo modelo inspirado por Perkins y proyecto Cero de la Universidad de Harvard (Stone, 1998). Ambos modelos apuntan a ayudar al aprendiz a reconocer los factores requeridos para que él/ella puedan lograr desempeñarse exitosamente en el contexto escolar. Las propuestas se desarrollan con la ayuda de investigaciones dirigidas para proveer procesos de gestión de la tarea, autogestión y negociación con el otro en el trabajo colaborativo, siempre alrededor de secuencias para facilitar la comprensión sobre el conocimiento, los métodos, los propósitos, y las formas de aproximación a ese conocimiento.

\section{Dentro del marco de la teoría de la actividad}

Este modelo está enmarcado dentro del constructivismo social de Vygotsky. En la teoría de la actividad se entenderá por actividad aquel conjunto de acciones que presentan una motivación intrínseca para el sujeto, lo que le lleva a buscar desarrollarlas activamente; en otras palabras, cuando un aprendiz disfruta el aprender por sí mismo, sin que le preocupen los requerimientos externos que le exigen el asumir ese proceso, y que en cambio, esas acciones lo estimulan en su deseo de comprender y asimilar el conocimiento implícito en esa determinada disciplina, las abordará como 'la actividad' razón de su hacer. La actividad en nuestra experiencia se ha dirigido a potenciar dinámicas de interacción en las zonas de desarrollo que está próximo a adquirir el aprendiz quién es quien las realiza a través de los diferentes procesos de interacción. Esta actividad se apoya estrechamente de procesos de regulación y autorregulación para lograr autonomía en el aprendiz sobre: la conciencia de lo que va aprender, a través de la comunicación de los objetivos; el dominio sobre lo que quiere comprender, intercambiando la anticipación operacional y planificación de la acción que va a ejecutar; y la apropiación de lo que quiere asimilar, argumentando los criterios funcionales y procedimentales acerca de lo que va a aplicar (Talízina, 1988). 


\section{Cómo nos consolidamos}

En 1993 las investigaciones se orientan a la educación básica y media, mediante el aporte de estrategias significativas para la utilización de tecnologías de información y comunicaciones en los procesos formales del aula de clase.

La línea, en la actualidad, es un grupo consolidado, líder en Antioquia y el país por su capacidad investigativa y aplicación de estrategias, resultado de las investigaciones realizadas y del apoyo internacional de entidades con quienes se han formalizado convenios de cooperación. Con el fin de mantener su liderazgo, la Línea se ha concentrado en la investigación de las diversas tecnologías de información y comunicaciones y su aplicabilidad en la educación, en todos los niveles, yen aportar al desarrollo de las comunidades, alternativas viables que les permita desarrollarse y participar de manera activa como miembros de la aldea global.

Desde sus inicios, el grupo tiene como visión: el fortalecimiento y profesionalización permanente del recurso humano del equipo de investigadores, la activa participación en eventos nacionales e internacionales de carácter investigativo, la política de cooperación entre grupos nacionales e internacionales para la investigación y desarrollo en Informática Educativa. La línea espera mantener el liderazgo en el desarrollo de investigaciones aplicadas en las áreas que apoyan los procesos de educación formal, llegando a obtener una cobertura de la educación básica, media, media técnica y superior y, adicionalmente mantener su participación en los espacios de reflexión relacionados con el tema, tanto en el contexto nacional como internacional.

El trabajo investigativo, como eje central de la actividad del grupo interdisciplinario, y los resultados obtenidos en las diferentes áreas de investigación, ha llevado al equipo de investigadores a obtener algunos reconocimientos y premios importantes, tales como: Proyecto innovador, orientado a una sensibilización y formación para un futuro sostenible, reconocimiento hecho al Proyecto Conexiones por la Organización de las Naciones Unidas para la Educación, la Ciencia y la Cultura (Unesco); Grupo de Excelencia en Investigación, premio otorgado por Colciencias en 1997; en 1998 la Línea fue escalonada como categoría B por Colciencias y el más reciente, Premio Anual de Investigación Universidad Eafit, conferido por la Universidad Eafit en 1999. Finalistas en el Stockholm Challenge Award de Suecia, categoría educación, en el año 2000 con el Modelo Conexiones e igualmente finalistas en el Junior Chellenge Award de Roma en el mismo año. Finalistas en el año 2001, categoría Global Village del Stockholm Challenge Award de Suecia, con el proyecto Escuela Global: Centro de Informática Veredal.

\section{Nuestra experiencia}

Los proyectos de investigación, iderados por el grupo de investigadores de la Línea de investigación y desarrollo en informática educativa, se han inscrito en las siguientes áreas de investigación: sistemas tutoriales, inteligencia artificial, multimedia e hipermedia, telemática educativa y comunidades educativas.

A continuación, de describen cada una de las áreas de investigación y los proyectos asociados. 


\section{- Sistemas tutoriales}

Esta área ha buscado promover la investigación sobre el potencial de las tecnologías de información y comunicaciones y su aplicabilidad en los procesos formales de educación superior, con el objetivo de encontrar la manera apropiada de incorporar estas tecnologías a los procesos de enseñanza y de proveer a los alumnos alternativas variadas de descubrir e interiorizar nuevos conocimientos.

\section{Cursos asistidos por computador}

El primer sistema tutorial que se desarrolla en esta área es el "Curso de Estática Asistido por Computador", utilizado aún por los profesores y estudiantes de los primeros semestres de la facultad de ingeniería civil, como apoyo a las cátedras y los laboratorios de ingeniería, y que llevó a la formalización de la Línea de investigación y desarrollo en informática educativa de la Universidad Eafit y a la creación de una línea de producción de cursos asistidos por computador, juegos y simuladores como material de apoyo a la docencia, llamada Línea Tutor.

\section{- Inteligencia artificial}

En esta área se inicia el desarrollo de sistemas tutoria!es inteligentes, con el objetivo de analizar su potencialidad como apoyo a los alumnos que cursan las primeras materias de matemáticas para ingenieros, centrándose en el tema de análisis y trazado de curvas y bajo la asesoría del doctor Gilles Imbeau, de la Universidad de Québec, Canadá.

Apolonio 1+: STI para análisis y trazado de curvas polinomiales El proyecto Apolonio 1+, financiado por la Universidad Eafit, desarrolló un prototipo de sistema tutorial inteligente (STI), cuyo objetivo fue propiciar, en los estudiantes de primer semestre del curso de matemáticas I, el desarrollo de la habilidad de solución de problemas.

Apolonio 1 + integró cuatro componentes, módulos o actores: Currículo, planificador, tutor e interfaz. Esta arquitectura, está basada en el modelo teórico del grupo Heron de la Universidad de Québec en Montreal.

Con Apolonio 1 + se trató de disminuir el tiempo y los costos de capacitación y aumentar la efectividad del aprendizaje, ya que se entrenó a la persona particular, aprovechando sus conocimientos previos en el tema a tratar y ofreciéndole una forma agradable de adquirir los nuevos conocimientos.

De esta investigación se obtiene una estructura de STI, que pretende lograr objetivos desde la conceptualización hasta la aplicación de los temas, la cual es aplicada mediante un proceso formal de evaluación del sistema a un grupo de estudiantes de la Universidad, y contrastado con un grupo control que no es sometido al tratamiento.

Los resultados obtenidos con este sistema fueron muy satisfactorios para los alumnos que fueron sometidos al tratamiento, e igualmente para el equipo de investigadores de la Línea de informática educativa, quienes a partir de estos resultados inician investigaciones complementarias en STI, con miras a mejorar el sistema en su Interactividad con el usuario y mejorar la eficiencia de los sistemas expertos que orientan y validan la información entregada al sistema. 


\section{- Multimedia e hipermedia}

Los proyectos desarrollados dentro de esta área de investigación buscaron implementar e integrar al ámbito educativo herramientas para multimedia e hipermedia, que ofrecieran una ayuda interactiva al estudiante durante el proceso de aprendizaje dirigido en la búsqueda de logros de objetivos de carácter pedagógico, con el fin de profundizar en estrategias de enseñanza y aprendizaje.

\section{Proyecto Merlín}

En 1990 se formalizó el estudio de las posibilidades de multimedia y telecomunicaciones aplicadas en educación con el Proyecto Merlín. El objetivo principal de este proyecto fue crear un laboratorio de investigación que integrara las áreas de multimedia e informática educativa en el proceso de adopción, desarrollo y transferencia de tecnologías que sirviera de apoyo al proceso de enseñanza-aprendizaje. Este proyecto fue financiado por la Universidad EAFIT, y obtuvo como resultado un laboratorio para desarrollo de aplicaciones multimedia a disposición de la comunidad educativa de la Universidad.

\section{Athena Muse 2 (AM2)}

Durante 1993 a 1995, representantes de la Línea I+D en informática educativa de la Universidad Eafit, participaron en el CECl (Centerfor Education on Computer Initiatives) del MIT en el proyecto denominado Athena Muse 2, con el fin de desarrollar un sistema para la producción de materiales multimedia e hipermedia en ambientes distribuidos. Igualmente, como aporte a la transferencia tecnológica desde este proyecto hacia la Universidad, se desarrolló un proyecto homólogo (Proyecto Homólogo Athena Muse 2) en la Línea de informática educativa, que permitió capacitar investigadores, transferir modelos de investigación y desarrollo, y mantener los vínculos de cooperación y transferencia tecnológica con el $\mathrm{CECl}$. Los resultados del proyecto genérico y la participación de la Universidad pueden apreciarse en la dirección http:// www-ceci.mit edu/am2/sponsor html

\section{- Telemática educativa}

Esta área tiene como objetivo analizar el efecto de la utilización de redes electrónicas de comunicación en los ambientes de aprendizaje escolar.

\section{Conexred}

El objetivo de este proyecto fue diseñar una infraestructura telemática apropiada y a bajo costo para soportar la conectividad de instituciones educativas de nivel básico y medio, con miras a mostrar de una forma muy práctica la factibilidad de establecer en las instituciones educativas redes de comunicaciones a costos abordables parlas mismas. Este proyecto fue financiado por la Fundación Carona y la Universidad Eafit en su primera fase de investigación e implementación posteriormente, el desarrollo del proyecto Conexiones fue financiado por Colciencias e Info/Dev Banco Mundial y las universidades Eafit y Pontificia Bolivariana.

La red interescolar, Conexred, fue desarrollada en el interior del Proyecto Conexiones, convirtiéndose en parte fundamental de su plataforma telemática (ver área Ambientes Tecnológicos de Aprendizaje), y actualmente conecta a más de 60 instituciones 
educativas de Antioquia y Santander a través de un servicio de acceso remoto. Esta red soporta la comunicación interinstitucional, permite la transferencia de archivos, el acceso a Internet, servicio de noticias y listas de distribución y apoya los procesos de soporte en línea y acceso a la información, componentes del Proyecto Conexiones.

\section{- Ambientes virtuales colaborativos}

El objetivo de esta área es investigar y desarrollar ambientes virtuales que permitan el aprovechamiento de herramientas de comunicación y productividad; el intercambio de información, recursos gráficos y objetos tridimensionales, facilitando la integración de las TIC's en los procesos formales de enseñanza-aprendizaje.

\section{Ambientes virtuales colaborativos aplicados a la educación superior}

Mediante este proyecto se construyó un sistema que permitiera la interacción de varias personas, separadas geográficamente, en un entorno virtual, como herramienta de soporte en un proceso de educación a distancia.

Para dicho proyecto, se plantearon tres fases progresivas, en las cuales se construyó una herramienta de trabajo colaborativo cada vez más interactiva. La primera consistió en un entorno virtual basado en texto, en el cual varias personas podían intercambiar mensajes escritos en un ambiente controlado por un profesor. En las siguientes fases se incluyó una interfaz de realidad virtual, que permitía (inicialmente, a un profesor y un alumno, y en la última etapa a varios alumnos al tiempo) recorrer conjuntamente un mismo entorno virtual e intercambiar algunos recursos básicos (como gráficas y objetos tridimensionales), a través de elementos virtuales de uso pedagógico. El impacto de dichas herramientas fue evaluado en un conjunto de sesiones de tutoría de un curso piloto de cultura ambiental, simulando un entorno de educación a distancia. Este proyecto fue financiado por Colciencias y la Universidad Eafit en su primera fase entre 1997 y 1998. Ver http://sigma.eafit.edu.co/vi rtualc

\section{- Ambientes tecnológicos de aprendizaje}

Esta área busca investigar y desarrollar ambientes tecnológicos de aprendizaje, donde se integren las tecnologías de información y comunicaciones al servicio de la educación formal.

\section{Conexiones: un ambiente tecnológico escolar}

A partir de 1995, Conexiones como proyecto en informática educativa, es desarrollado por la Universidad Eafit en convenio con la Universidad Pontificia Bolivariana. Conexiones propone la creación de ambientes de aprendizaje colaborativos, donde se incorporen las tecnologías de información y comunicaciones al currículo de la educación básica. Conexiones ha permitido analizar el efecto de la utilización de redes interescolares, interfaces gráficas de comunicaciones, herramientas de productividad, software educativo y proyectos colaborativos en el aula de clase. En especial, el proyecto de investigación ha evaluado: su impacto en la formación integral de estudiantes entre los 7 y los 16 años ( $2^{\circ}$ a $9^{\circ}$ grado); el fortalecimiento de valores sociales y culturales; la sistematización de información; el trabajo por proyectos y el aprendizaje cooperativo, entre otros.

Hoy en día, la Línea de I+D en informática educativa concentra sus esfuerzos de investigación en el desarrollo del proyecto Conexiones, que recoge investigaciones 
particulares en cada una de las áreas de investigación, convirtiéndose en el proyecto líder de la Línea.

En Conexiones están vinculadas, actualmente, más de 60 instituciones educativas de diferentes regiones del país, quienes están interconectadas entre si a través de la red interescolar Conexred (ver área telemática educativa). El Proyecto consta con una participación de 6000 alumnos, quienes junto a docentes y directivas reciben capacitación, soporte técnico y pedagógico, brindado por Conexiones durante todo el proceso de incorporación de las NTCl al aula.

En sus diferentes etapas, este proyecto ha recibido apoyo de organismos nacionales e internacionales como: la Fundación Corona, Colciencias, la Secretaría de Educación Municipal de Medellín (Edúcame), la Secretaría de Educación Departamental - Antioquia (Seduca), el Centro de Ciencia y Tecnología de Antioquia, el programa Infodev del Banco Mundial, la Unesco y las universidades Eafit y Pontificia Bolivariana. Ver http://www. conexiones.eafit.edu.co

\section{Entendiendo las ciencias con mapas conceptuales}

Con este proyecto se pretende aprovechar las herramientas computacionales como facilitadores del proceso da representación y almacenamiento de contenidos de tipo científico, haciendo uso de la estrategia de mapas conceptuales y de metodologías para la adquisición del conocimiento. Se espera desarrollar un atlas de ciencias que incorpore los conceptos de las ciencias representados en mapas conceptuales a través de la herramienta computacional Cmaps, desarrollada por el Instituto de Cognición Humanada la Universidad de West, Florida, quedando una base de mapas digitales al servicio de estudiantes y docentes de todos los niveles educativos.

\section{- Ambientes virtuales de aprendizaje}

Dentro de esta línea los mayores esfuerzos se han concentrado en la investigación sobre la mediación tecnológica de los procesos académicos, educativos y de capacitación, donde las herramientas computacionales soportan actividades en un esquema de ambiente de aprendizaje que van desde lo virtual a lo bimodal, haciendo éntasis en el desarrollo de competencias básicas y la aplicación de estrategias didácticas propias de estos entornos.

\section{IE TOOL}

Herramienta computacional diseñada e implementada como mediador tecnológico de procesos educativos formales para potenciar el desarrollo de habilidades y competencias de tipo argumentativo. La investigación tiene como finalidad mejorar la comprensión de los procesos educativos que demanda el trabajo con las TICs, relacionados especialmente, con la interacción entre las personas y con los materiales didácticos. Se quiere indagar qué acciones de tipo didáctico son necesarias tener en cuenta, y aplicar, para optimizar algunos de los aspectos en los que las TICs representan cambios importantes, como el hecho de la enorme reducción en los costos de almacenaje y procesamiento de la información, de que se hayan ampliado los accesos a los recursos de aprendizaje o de que se faciliten a través de ellos los aprendizajes de tipo autónomo. Esta herramienta forma parte de una investigación en didáctica de las ciencias y se espera medir el impacto de la mediación tecnológica en procesos de educación formal a nivel universitario. 


\section{- Comunidades educativas}

Como resultado del modelo para la incorporación de tecnologías en los ambiente de aprendizaje, modelo Conexiones, se ha iniciado una nueva etapa de investigación orientada por el lema: "de la escuela a la comunidad", donde se busca modelar la situación educativa de las comunidades rurales, mediante la creación de centros tecnológicos comunitarios en las escuelas y colegios que han logrado apropiare! modelo Conexiones, como una propuesta global a nivel institucional.

\section{Conexiones II, escuela global: de la escuela a la comunidad}

Esta investigación pretende desarrollar un modelo pedagógico y de gestión para orientar la implementación y sostenibilidad de centros tecnológicos educativos de acceso comunitario, aprovechando las experiencias obtenidas con la incorporación del modelo Conexiones en centros educativos rurales y zonas marginadas, y las experiencias en la puesta en marcha del Centro de Informática Veredal El Tablazo, Municipio de RionegroAntioquia. Como resultado parcial de esta investigación se ha podido observar cómo, a partir de la experiencia de apropiación del modelo Conexiones en los centros educativos rurales, ha sido posible captar el interés de las comunidades para mejorar su calidad de vida, sus posibilidades de empleo y desarrollo cognitivo, haciéndolas participes de la experiencia de diseñar, y poner en marcha un proyecto de beneficio comunitario, donde prevalece la búsqueda del desarrollo social, a partir del compromiso, la participación, las acciones educativas y la mejora en los procesos comunicativos entre padres e hijos, escuela y comunidad, fundamentándose en el uso significativo de las tecnologías de información y comunicaciones como herramienta de trabajo.

\section{Conclusiones}

Como se ha indicado, los procesos de investigación de la Línea I+D en informática educativa, de la Universidad Eafit, ha tenido como finalidad mejorar la comprensión de los procesos educativos que demanda el trabajo con las TIC, relacionados especialmente, con la interacción entre las personas y los materiales didácticos. Se ha querido indagar qué acciones de tipo didáctico son necesarias a tener en cuenta, y aplicar, para optimizar algunos de los aspectos en los que las TIC representan cambios significativos.

Estamos de acuerdo con Castells (2001) cuando dice que la demanda que se hace para construir o desarrollar las habilidades necesarias para vivir y trabajar en la nueva sociedad de la información, requiere investigaciones comprometidas acerca de como aproximar las TIC a los procesos educativos. Ello ha implicado crear formas consistentes de interacción a través de estos medios, entre quienes aprenden, quienes enseñamos o acompañamos los procesos y los materiales que puede el aprendiz encontrar a su disposición.

De acuerdo con las acciones de moni-toreo y evaluaciones llevadas a cabo en nuestros proyectos, se han llegado a establecer entre otros los siguientes indicadores a tener en cuenta:

1. Las tecnologías de información y comunicaciones no constituyen por sí mismas un beneficio educativo; para ello, es preciso que se integren en un proyecto pedagógico con sentido. 
2. La incorporación de las tecnologías de información y comunicaciones tiende a desorganizar la estructura del ambiente de aprendizaje tradicional y las estructuras organizativas de la institución. Esto exige la existencia de un proyecto institucional que indique un norte y que involucre docentes, directivos y administrativos por igual.

3. La propuesta de un modelo pedagógico coherente, que incorpore las tecnologías de información y comunicaciones, con un enfoque fundamentalmente socioconstructivista, trabajo por proyectos y aprendizaje colaborativo, facilita la innovación educativa con tecnologías en los ambientes de aprendizaje.

4. Los proyectos de informática educativa están produciendo cambios en: (1) la formada entender y llevar la intervención docente, de una posición vertical de "enseñante" a una más horizontal de "facilitador y orientador" del aprendizaje, y (2) los estilos docentes que se encaminan hacia el abordaje del proceso de enseñanza y aprendizaje como cuerpo colegiado y no como profesores aislados.

5. Los usos pertinentes de las tecnologías de información y comunicaciones, con temas cercanos a las experiencias de vida de los usuarios permite potenciar y aumentar los logros pedagógicos.

6. Las estrategias colaborativas y de comunicación telemática, permiten mejorar el autoconcepto, la motivación, las actitudes de tolerancia y la cooperación.

\section{Bibliografía}

Atuesta M. del R.; Sierra, SE. y Zea C. (1993) "Apolonio 1+: Una Alternativa para el sistema educativo colombiano". X Encuentro Universitario de Informática Proceedings. ASCUN, Externado de Colombia University.

Atuesta M. del R, SILVIA, S. y ZEA C. (1994) "Apolonio 1+ Evaluación Experimental de un sistema tutorial inteligente'. II Congreso Iberoamericano de Informática Educativa. Proceedings, Lisboa-Portugal.

Castells, M. (2001) L' Educació a la Societat de la informació. Barcelona, Ponencia introductoria del "Foro L'Escola i la Ciutat".

Gagné, R. M. (1971) Las condiciones del aprendizaje. Madrid, Ed. Aguilar (Ed. Original: "The conditions of learning". New York, Holt, Hinehart \& Wiston, 1965).

Gardner, H.; Sternberg, R.; Krechevsky, M.; Okagaky, L. (1996) "Intelligence in Context: Enhancing Students" Practical Intelligence for Schools", en: Classsroom lessons. Integrating Cognitive Theory and Classroom Practice. Cambridge, Bradford Book, MIT Press.

González, M. y Zea, C. (1997) The path to future directions of colombian interschool communication networking. Conference on Global Knowledge Proceedings, TorontoCanadá. Juno 23-25.

González, M. y Zea, C. (1997) Proyecto Conexiones: Desafíos y estrategias. Conference on Knowledge Global 97 Proceedings, Bogota-Colombia. 
Imbeau, G. y Zea, C. (1991) Building a Course Curriculum that Supports an Intelligent Learning Environment, IV International Symposium on Artificial Intelligence. Proceedings. Cancun-Mexico.

Piaget, J. (1978) La equilibración de las estructuras cognitivas. Madrid, Siglo XXI, 1978.

Solórzano, B. y Zea, C. (1992) Nuevas tendencias Informáticas y sus posibilidades en los sistemas educativos Multimedios e Hipermedios. I Congreso Iberoamericano de Informática Educativa Proceedings. Santo Domingo-Dominican Republic.

Solórzano, B., Zea, C. et al. (1993) MERLIN: Un proyecto de multimedia para abrir fronteras. $X$ Encuentro Universitario de Informática Proceedings. ASCUN, Externado de Colombia University.

Stone, M. (editor) (1998) Teaching for Understanding. San Francisco, Jossey Bass Publishers.

Talízina, N. F. (1988) Psicología de la enseñanza. Moscú, Editorial Progreso, 1988.

Trujillo, J.; Zea, C. y González, M. (1998). Conexiones, Building a Technological Learning environment. ED. MEDIA 98 Proceedings. Germany, July.

Trujillo, J. y Zea, C. (1994) Colombian Interschool Communications Network. TELED'94 Proceedings, New Mexico USA.

Trujillo, J. y Zea, C..( 1994) Las tecnologías en la Educación. II Congreso Iberoamericano de Informática Educativa. Proceedings, Lisboa-Portugal.

Trujillo, J. El modelo de aprendizaje de Ausubel como soporte teórico para conceptualizar la teletutorización, (en prensa).

Uribe, M. V.; Yepes, Y. y Zea, C. (1995) Consideraciones para el diseño de Interfaces humano-computador. Informática al día, Journal, Colombia.

Zea, C. y Vélez, A. M. (1999) "El Desarrollo de la lúdica alrededor de los ambientes de aprendizaje propuestos por Conexiones". El juego y la lúdica: Alternativas de desarrollo social para el siglo XXI. International Seminar.

Zea, C; Vélez, A. M. y Atuesta, M. Del H. (1999) "Conexiones: Una red virtual de ambientes de aprendizaje colaborativos" $1^{a}$. Jornada Nacional de Tecnología y Educación. La Paz, Bolivia, June.

Zea, C. et al. Sistemas Hipermedios Colaborativos. Nuevos ambientes de aprendizaje. IV Congreso Iberoamericano de Informática Educativa. Proceedings. Brasilia-Brasil. Oct. de 1998

Zea, C. (1998). Proyecto Conexiones: Educación para un futuro sostenible. I Taller Encuentro de las Américas. Proceedings. Banco Mundial. Washington-USA, Juno.

Zea, C. (1997). Retos y estrategias del Proyecto Conexiones. Educación y Tecnología: la relación que usted nunca imagino. Seminar. Bogotá, Colombia. Doc. 
Zea, Claudia of al. (1996) Conexiones an Interactive Learning Envíronment. World Conference on Educational Multimedia and Hypermedia - ED-MEDIA 96 Proceedings, Boston-USA., Juno 17-22.

Zea, C. (1994). Ambientes informáticos para el aprendizaje. I Encuentro de Informática Educativa. Proceedings, Madrid-Spain.

Zea, C.; González, M.; Atuesta, M. del R. Modelo de incorporación de tecnologías de información y comunicaciones, implementado en la red interescolar del proyecto Conexiones. Cátedra de Educación. Medellín, Universidad Nacional, Proceedings, 1998.

Zea, C.; González, M.; Atuesta, M. del H. (1998) Sistemas Hipermedios Colaborativos: Nuevos Ambientes de Aprendizaje. Congreso Colombiano de Informática Educativa. Proceedings. Manizales, Colombia.

Zea, C. y Trujillo, J. (1994) Redes Interescolares de Comunicaciones. II Congreso Colombiano de Informática Educativa Proceedings, Cali - Colombia - and II Foro Colombiano de investigadores en Informática Educativa. Medellín - Colombia.

Zea C. (1996) Conexiones: Un ambiente de aprendizaje colaborativo basado en tecnologías de información y comunicaciones. Systems Journal, ACIS, Colombia.

Zea, C. (1995) Conexiones: Un ambiente de aprendizaje con tecnologías informáticas. Informática al día Journal, Colombia.

Zea, C. y otros. (1995) Estructuración de ambientes de aprendizaje con Multimedia. Informática al día Journal, Colombia.

Zea, C. y Solórzano, B.( 1993). Hipermedia y Educación. Systems Journal, ACIS, Colombia.

Zea, C.; Saldarriaga, O. y Turizo, Y. (1993). Consideraciones acerca de Multimedia. Informática al día Journal, Colombia.

Zea, C. y Solórzano. (1993) Hipermedios: una nueva forma de representación y manejo de la información. Informática al día Journal, Colombia.

Zea, O. y Solórzano, B. (1991) Apolonio 1+: Sistema Tutorial Inteligente para la Solución de Problemas en Matemáticas. EAFIT University Journal, No. 83, Colombia. 